\title{
Lower Urinary Tract Symptoms in Myasthenia Gravis
}

\author{
Fuyuki Tateno $^{a} \quad$ Ryuji Sakakibara ${ }^{a, b}$ Yosuke Aiba $^{a}$ \\ aNeurology, Internal Medicine, Sakura Medical Center, Toho University, Sakura, Japan; \\ bNeurology, Chiba University, Chiba, Japan
}

\section{Keywords}

Myasthenia gravis · Lower urinary tract symptom · Quality of life · Overactive bladder

\begin{abstract}
It remains uncertain to what extent lower urinary tract (LUT) symptom (LUTS) is a comorbidity of myasthenia gravis (MG). We prospectively administered a LUTS questionnaire devised for detecting neurogenic pelvic organ dysfunction (not validated) in an MG group and a healthy control group and compared the results. The MG group comprised 21 patients: 15 women and 6 men, with age range 22-73 (mean 47) years, illness duration range 0.2-8 (mean 3.5) years, median Myasthenia Gravis Foundation of America (MGFA) grade 2, all walking independently. Therapies included thymectomy in 17 , predonisolone $5-20 \mathrm{mg} /$ day in 10 , and pyridostigmine bromide 60-180 mg/day in 9 patients. The control group, who were undergoing an annual health survey, comprised 235 consecutive subjects: 120 women and 115 men, with age range 30-69 (mean 48) years. The questionnaire had 9 questions. Each question was scored from 0 (none) to 3 (severe) with an additional quality of life (QOL) index scored from 0 (satisfied) to 3 (extremely dissatisfied). Statistical analysis was made using Student's $t$ test. Compared with the control subjects, the frequency of LUTSs in the MG patients was significantly higher for daytime frequency $(43 \% ; p<0.01)$, nocturia (24\%; $p<0.01)$, and urinary incontinence (43\%; $p<0.05)$. The LUTS-related QOL index for the MG patients was significantly higher for MG patients as a whole than that for all control patients $(29 \%)(p<0.05)$. In conclusion, our study results showed that MG patients had significantly more LUTSs (overactive bladder) than healthy control subjects and had worse LUTS-related QOL; therefore, amelioration of LUTS in MG is important.
\end{abstract}

\section{Karger $\stackrel{2}{\circ}$}




\section{Introduction}

Myasthenia gravis (MG) is a rare autoimmune disorder of the neuromuscular junction [1]. The estimated prevalence of MG is approximately 20 cases per 100,000 population, with the disease affecting twice as many women as men. The overall prevalence is approximately 150-200 per million. Around $70 \%$ of the MG patients are seropositive for antibodies against the nicotinic acetylcholine ( $\mathrm{ACh}$ ) receptors (nAChRs) found in the postjunctional membrane of the striated muscles (the remaining MG patients are seronegative; some of them have antibodies against muscle-specific kinase) [1]. Although the muscles directly associated with the lower urinary tract (LUT) are smooth muscles, they are further surrounded by the striated muscles of the external sphincter and the pelvic floor. LUT symptoms (LUTSs) have been reported in case reports of MG, varying from overactive bladder and urinary incontinence [2, 3] to retention [4]. However, it remains uncertain to what extent MG pathology contributes to the LUTS. This is especially difficult to untangle in older MG patients, since LUTS, particularly voiding dysfunction, is also common in older men due to prostatic hyperplasia [5], lumbar spondylosis, diabetes, and overactive bladder due to white matter disease which is common in older men and women [6]. In order to begin to sort out this comorbidity issue, we performed a LUTS questionnaire in an MG group and a healthy control group and compared the results.

\section{Materials and Methods}

This was a questionnaire study. The inclusion criteria for the patients were those who had generalized MG and were able to walk independently to visit our clinic. We recruited 21 consecutive patients with MG in a year [1]: 15 women and 6 men, with age range 22-73 (mean 47) years, illness duration range 0.2-8 (mean 3.5) years, and Myasthenia Gravis Foundation of America (MGFA) quantitative score [1] 2 (mildly generalized) to 3 (moderately generalized) (median 2), all having weakness and fatigue in the ocular and limb muscles. Among 21 MG patients, the peak MGFA score was $3 \mathrm{~b}$ in 3, 3a in 7, and 2 in 11 . None had a history of artificial respiration/tracheostomy. All patients had shown waning of the compound muscle action potentials by a repetitive nerve stimulation test and increased serum antibodies against $\mathrm{nAChR}(0.3-855 \mathrm{nmol} / \mathrm{L}$, normal $<0.3 \mathrm{nmol} / \mathrm{L})$. Therapies included extended thymectomy in 17, steroid pulse therapy with plasmapheresis in one, prednisolone 5-20 mg/ day in 10 , tacrolimus $3 \mathrm{mg} /$ day in 3, and pyridostigmine bromide $60-180 \mathrm{mg} /$ day in 9 patients.

The inclusion criteria for the controls were local individuals who participated in an annual health survey. We recruited 235 consecutive subjects in a year: 120 women and 115 men, with age range 30-69 (mean 48) years. All participants were checked for medical risk factors and physical status, and all underwent a chest X-ray, blood chemistry test, and urinalysis.

The exclusion criteria for both healthy subjects and MG patients were those with a known risk factor for LUT dysfunction, such as diabetes (excluded number of subjects; 16 controls and no MG); those who had a previous history of pelvic organ prolapse (no controls and no $\mathrm{MG}$ ); those who were taking anticholinergics, alpha-blockers, or beta-stimulants that might affect LUT function (no controls and no MG); and males who had a history of benign prostatic hyperplasia (7 controls and no MG).

The female-to-male ratio in our MG patients was slightly higher than that in the control group, but this difference did not reach statistical significance ( $p=0.073, \chi^{2}$ test). In both groups, we administered a LUTS questionnaire that we devised for detecting neurogenic 
pelvic organ dysfunction [7]. After providing informed consent, each participant was handed a questionnaire sheet by a medical or co-medical staff member. The questionnaire had 9 questions consisting of 4 questions on the filling phase and 5 on the voiding phase. Each question was scored on a scale of 0 (none), 1 (>once a month; mild), 2 (>once a week; moderate), or 3 (>once a day; severe). Additional questions were included on overall subjective satisfaction about bladder condition. These were scored from 0 (satisfied), 1 (mildly dissatisfied), 2 (moderately dissatisfied), to 3 (extremely dissatisfied) to yield a quality of life (QOL) index. The questionnaire required $<10 \mathrm{~min}$ to complete (Table 1). In either group, if any question was answered with a score other than 0 , it was counted as the presence of a symptom. Student's $t$ test was used for the statistical analysis. This study was made according to the Declaration of Helsinki. All participants gave informed consent before participating in the study.

\section{Results}

Compared with the control subjects, the frequency of LUTSs in the MG patients was significantly higher for daytime frequency ( $>8$ times) (43 vs. $0 \% ; p<0.01$ ), nocturia (more than twice) (24 vs. $6 \% ; p<0.01$ ), and urinary incontinence (43 vs. $21 \% ; p<0.05$ ) (Table 2). With regard to the subtypes of urinary incontinence, the frequencies of stress-type incontinence $(29 \%)$ and urgency-type incontinence $(14 \%)$ were higher in the patient group, but these differences did not reach statistical significance. The LUTS-related QOL index was significantly higher (indicating a worse QOL) for the MG patients (29 vs. 9\%; $p<0.05$ ) than for the controls. The majority of the MG patients with LUTS reported that LUTS had not appeared until after the onset of their motor disorder, and LUTS appeared and progressed along with motor disorder.

A comparison of the results by sex showed stress incontinence to be significantly more common in women $(p<0.01)$ among the controls. In the MG group, these differences did not reach statistical significance, although stress incontinence was noted only in women. A comparison of the results by MG severity (MGFA motor grades 2 and 3) showed that urinary incontinence was more common in the grade $3(n=17,83 \%)$ than the grade 2 patients $(n=$ $5,25 \%$ ), though these differences were not statistically significant. In the MG group, there was no relationship between LUTS and the peak MGFA score, history of thymectomy, duration of disease, nAChR antibody titer, or age. Urinary urgency and frequency were more common in the MG patients taking a cholinesterase inhibitor $(n=16,78 \%)$ than in those not taking this medication $(n=11,50 \%)$, and in the MG patients taking a corticosteroid $(n=17,82 \%)$ than in those not taking a corticosteroid $(n=9,44 \%)$, though these differences did not reach statistical significance.

\section{Discussion}

Thus far, as compared with diseases of the brain, spinal cord, and peripheral nerves [8, 9], little has been studied concerning the relationship between neuromuscular diseases/ myopathies and LUTS. Among the few existing studies, Dixon et al. [10] reported cases of stress urinary incontinence in limb-girdle muscular dystrophy and Dickson et al. [11] reported cases of myotonic dystrophy, both likely due to pelvic floor weakness. In MG, a variety of LUTS have been reported in case reports, from overactive bladder and urinary incontinence [2, 3] to retention [4] (Table 3). However, it remains uncertain to what extent MG contributes to the LUTS. To our knowledge, the present study is the first to show that compared with healthy

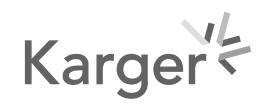




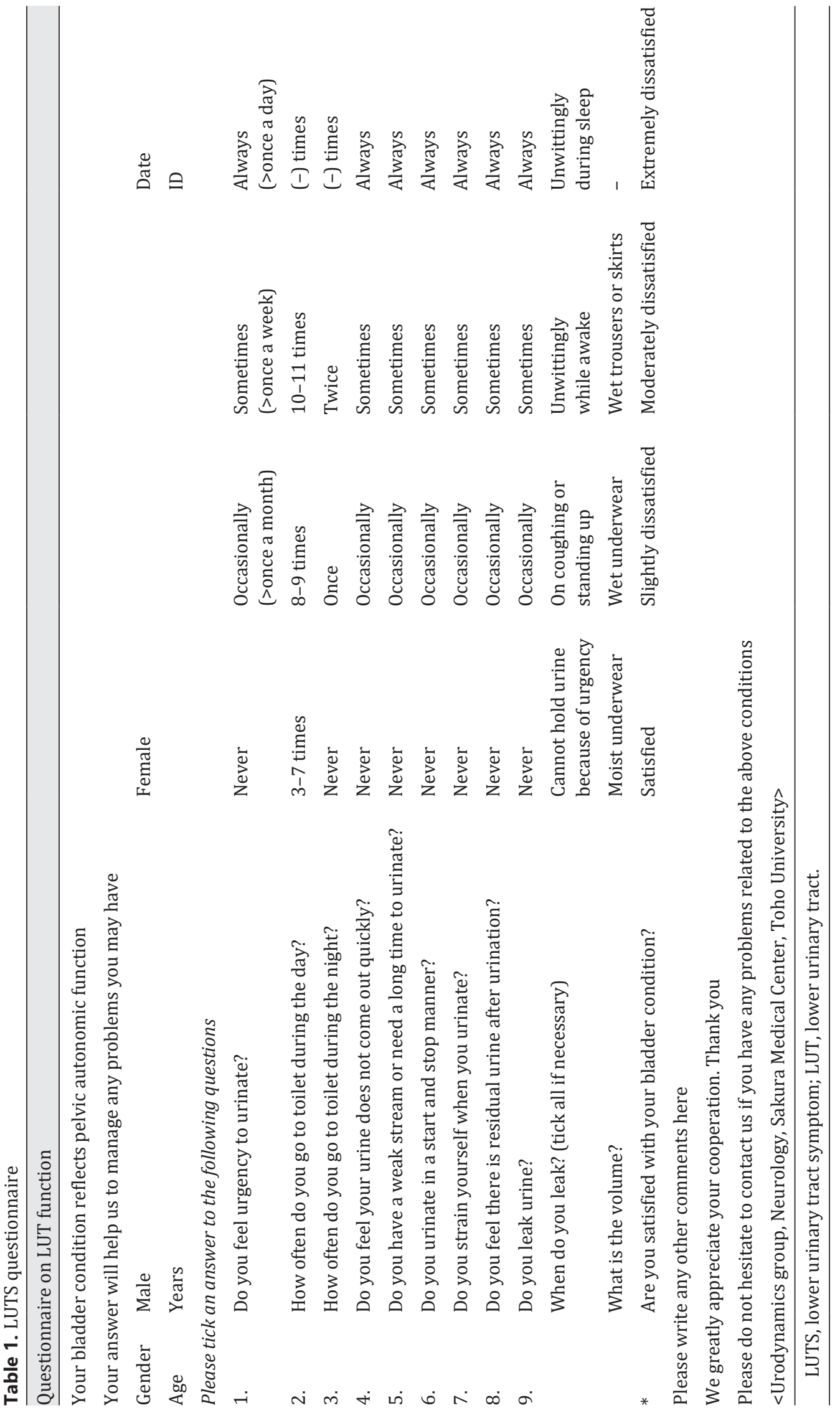




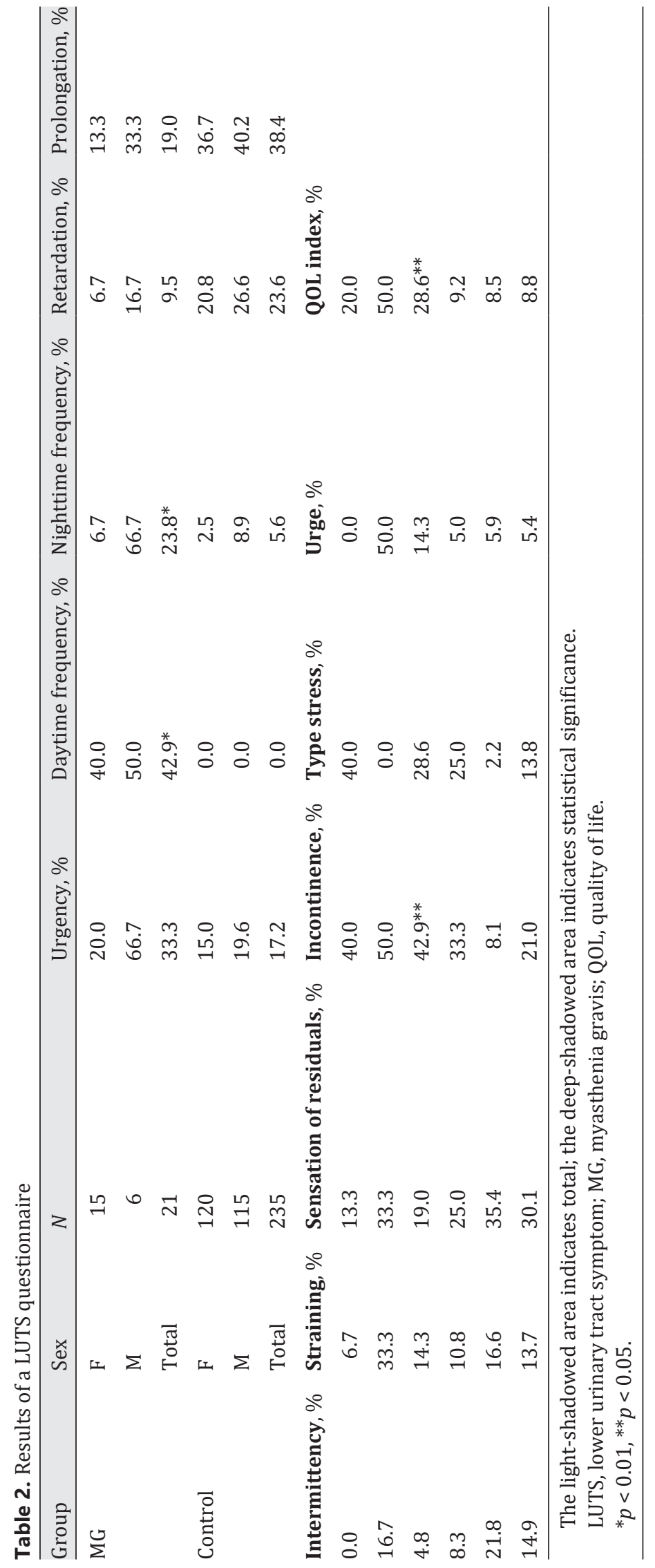

\section{Karger'}




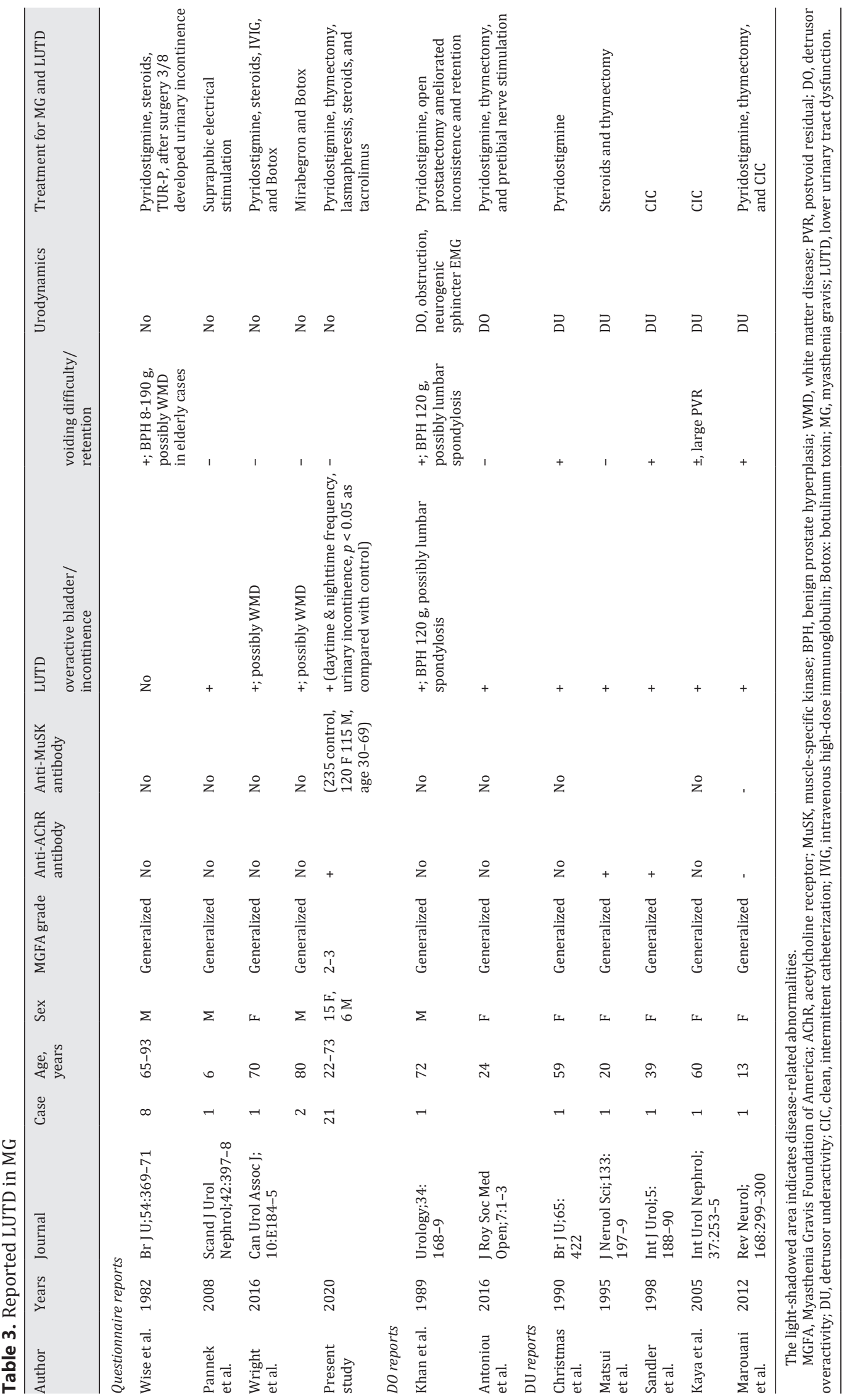


controls, MG patients have a statistically significant increase in daytime frequency $(43 \% ; p<$ $0.01)$, nocturia $(24 \% ; p<0.01)$, and urinary incontinence $(43 \% ; p<0.05)$, indicating impaired storage function in MG. Furthermore, these symptoms significantly increased a LUTS-related QOL index $(29 \% ; p<0.05)$, that is, they worsened QOL in MG patients compared to controls.

Since we did not perform urodynamics in any of our patients, we do not know the exact pathological mechanism explaining the above findings of the storage dysfunction. However, looking at the urodynamic reports in MG (Table 3) and neuro-urology reports [8, 9], there might be a direct cause and/or a secondary or associated cause producing LUTS in MG. Detrusor overactivity (DO; as seen in Table 1) is usually attributed to the brain/supra-sacral spinal cord lesions, but it might also originate from partial peripheral nerve lesion (by irritation and ephaptic transmission of the nerve fibers). Another possibility for DO is that pyridostigmine, commonly prescribed to ameliorate muscular weakness in MG, stimulates bladder smooth muscles enough to generate DO [12]. In our previous study [13], a 42-year-old man with MG (MGFA2, under $120 \mathrm{mg}$ /day pyridostigmine) showed DO and small bladder capacity $(150 \mathrm{~mL})$ on urodynamics. He had no brain/spinal cord diseases. Since anticholinergics are contraindicated for $\mathrm{MG}$, we started him on $100 \mathrm{mg} /$ day milnaciplan, a selective serotonin-noradrenaline reuptake inhibitor. This medication ameliorated his OAB successfully (bladder capacity increased to $198 \mathrm{~mL}$ ) without worsening of MG [13]. Daytime urinary frequency $(p<0.05)$ and stress urinary incontinence in females, though not statistically significant in the present study, have been shown to be common in MG, presumably reflecting pelvic floor descent on straining/coughing by muscle weakness, as reported in muscular dystrophy [10], and possibly, weakness of the external urethral sphincter muscle that is innervated by both somatic and sympathetic nerves. Nocturia in our MG cases might derive from nocturnal polyuria, since many patients were taking oral predonisolone, and polyuria induced by cortisol has been described [14].

In contrast, while not statistically significant, our MG patients also had a trend of voiding dysfunction. While we did not perform urodynamics in any of our patients, detrusor underactivity (as seen in Table 1) might originate from peripheral nerve/detrusor smooth muscle lesions. There does exist an ACD2 gene mutation that leads to a rare congenital visceral myopathy [15], but such visceral myopathy is not part of MG, since muscarinic AChR (a sevenhelix G-protein-coupled membrane protein) is different from nAChR (a pentameric ligandgated ion channel). One possibility might be that MG can occur together with autoimmune autonomic neuropathy, which inhibits bladder smooth muscles through ganglionic nAChR blockade to further generate detrusor underactivity [16, 17]. A similar mechanism is speculated in Lambert-Eaton myasthenic syndrome (LUTSs were noted in 24\% of LEMS cases, but the study had no control group) [18].

The role of sphincter innervation on voiding dysfunction in MG has a debate. As described above, external sphincter muscles are innervated by both the somatic nerve (nAChR) and sympathetic nerve (alpha 1A/D adrenergic receptors). Sphincter obstruction in women is extremely uncommon, except for Fowler's syndrome. It is reported that a woman with Guillain-Barre syndrome produced urodynamic outlet obstruction. Guillain-Barre syndrome primarily affects large myelinated fibers, but pathology studies have revealed moderate to severe loss of small myelinated fibers by 2 mechanisms: one is peripheral nerve irritation or ephaptic transmission in the lumbosacral autonomic fibers, from either bystander inflammation or immune attack to the autonomic fibers; another is immune attack to the inhibitory spinal cord interneurons, which secondarily leads to lumbosacral autonomic hyperactivity [19]. Comorbid neuropathy in MG, as described above, might produce such sphincter obstruction by somatic and/or sympathetic partial denervation.

As seen in our MG patients, LUTS significantly affects patients' QOL $(p<0.05)$; therefore, LUT dysfunction can become an important therapeutic target. While often used

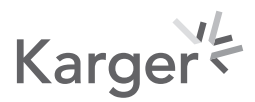


for management of overactive bladder (urinary urgency/frequency), anticholinergics are contraindicated for MG. Therefore, to treat overactive bladder in MG, serotonergic drugs (e.g., serotonin-noradrenaline reuptake inhibitors) [13] and selective adrenergic beta 3 receptor agonists [20] may be the best options. When stress urinary incontinence is proved by examination and tests, clenbuterol (an adrenergic beta 2 receptor agonist) [21] and mesh surgeries for counteracting pelvic floor descent are good choices. Again, urodynamics has a place, but clinical examination is still important to perform in all MG patients.

Limitations of the study obviously include the small number of patients. Therefore, falsenegative and false-positive (by chance) results cannot be excluded. In addition, limitations of the study include the use of a questionnaire that has not yet been validated. However, the LUTS questionnaire used here is the tool we devised for detecting neurogenic pelvic organ dysfunction. This tool proved useful to clearly differentiate LUTSs of the healthy population from LUTSs of neurodegenerative diseases (Parkinson's disease [7] and spinocerebellar ataxia 6 [22]) and psychogenic diseases (major depression [23]). Another limitation of the questionnaire we used is that, it was so sensitive (e.g., when someone has prolongation of only once or twice a month, it is counted) that the control group showed $38.4 \%$ of prolongation and $23.6 \%$ of retardation. This obviously needs care to be compared with other questionnaires. Limitations of the study also include the absence of urodynamics data. However, our results clarify that some MG patients have LUTSs that require medical attention. Therefore, a future study with a large number of patients is warranted. In conclusion, the results of the present study showed that MG patients had significantly more LUTSs (overactive bladder) and worse LUTS-related QOL than healthy control subjects; therefore, amelioration of LUTS is important.

\section{Acknowledgements}

We here appreciate collaboration given by Dr. Masahide Kashiwado and Dr. Koichi Kashiwado in Kashiwado Memorial Fund and the Kashiwado Hospital, Chiba, Japan.

\section{Statement of Ethics}

The present research complies with the guidelines for human studies, and the research was conducted ethically in accordance with the World Medical Association Declaration of Helsinki. The present research involves human subjects (patients). All the patients have given their written informed consent. The present research was approved by the Ethics Committee, Sakura Medical Center, Toho University (2011-059). Written informed consent for publication was obtained from all patients.

\section{Conflict of Interest Statement}

The authors have no conflicts of interest to declare.

\section{Funding Sources}

The authors have no funding sources to declare.

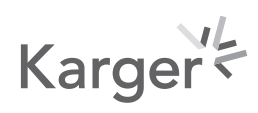


Tateno et al.: Lower Urinary Tract Symptom in Myasthenia Gravis

\section{Author Contributions}

F.T.: acquisition, analysis, and interpretation of data. R.S.: study concept and design, acquisition of subjects and/or data, analysis and interpretation of data, and preparation of the manuscript. Y.A.: acquisition of subjects and/or data.

\section{References}

1 Jaretzki A, Barohn RJ, Ernstoff RM, Kaminski HJ, Keesey JC, Penn AS, et al. Myasthenia gravis: recommendations for clinical research standards. Task force of the medical scientific advisory board of the myasthenia gravis foundation of America. Ann Thorac Surg. 2000;70:327-34.

2 Wright I, Civitarese A, Baverstock R. The use of intra-detrusor onabotulinumtoxinA in patients with myasthenia gravis. Can Urol Assoc J. 2016;10:E184-185.

3 Antoniou A, Mendez Rodrigues J, Comi N. Successful treatment of urodynamic detrusor over-activity in a young patient with myasthenia gravis using pretibial nerve stimulation with follow-up to two years. JRSM Open. 2016 Aug 1;7(8):2054270416653684.

4 Marouani I, Kraoua I, Benrhouma H, Rebai I, Rouissi A, Koubaa S, et al. Seronegative myasthenia gravis with bladder dysfunction. Rev Neurol. 2012;168:299-300.

5 Sakakibara R, Hamano S, Uchiyama T, Liu Z, Yamanishi T, Hattori T. Do BPH patients have neurogenic detrusor dysfunction? A uro-neurological assessment. Urol Int. 2005;74:44-50.

6 Sakakibara R, Panicker J, Fowler CJ, Tateno F, Kishi M, Tsuyusaki Y, et al. Is overactive bladder a brain disease? The pathophysiological role of cerebral white matter in the elderly. Int J Urol. 2014;21:33-8.

7 Sakakibara R, Shinotoh H, Uchiyama T, Sakuma M, Kashiwado M, Yoshiyama M, et al. Questionnaire-based assessment of pelvic organ dysfunction in Parkinson's disease. Auton Neurosci. 2001;92:76-85.

8 Panicker JN, Sakakibara R. Lower urinary tract and bowel dysfunction in neurologic disease. Continuum. 2020; 26:178-99.

9 de Groat WC, Griffiths D, Yoshimura N. Neural control of the lower urinary tract. Compr Physiol. 2015;5:32796.

10 Dixon PJ, Christmas TJ, Chapple CR. Stress incontinence due to pelvic floor muscle involvement in limb-girdle muscular dystrophy. Br J Urol. 1990;65:653-4.

11 Dickson MJ, Massiah N, Church E. Urinary stress incontinence as the presenting feature of myotonic dystrophy. J Obstet Gynaecol. 2012;32:102.

12 Obara K, Kobayashi Y, Chino D, Tanaka Y. Effects of distigmine on electrical field stimulation-induced contraction of mouse urinary bladder smooth muscles. Pharmacology. 2017;99(3-4):106-13.

13 Sakakibara R, Ito T, Uchiyama T, Awa Y, Yamaguchi C, Hattori T. Effects of milnacipran and paroxetine on overactive bladder due to neurologic diseases: a urodynamic assessment. Urol Int. 2008;81:335-9.

14 Joles JA, Rijnberk A, van den Brom WE, Dogterom J. Studies on the mechanism of polyuria induced by cortisol excess in the dog. Vet Q. 1980;2:199-205.

15 Rolle U, Puri P. Structural basis of voiding dysfunction in megacystis microcolon intestinal hypoperistalsis syndrome. J Pediatr Urol. 2006;2:277-84.

16 Muppidi S, Vernino S. Autoimmune autonomic failure. Handb Clin Neurol. 2013;117:321-7.

17 Miglis MG, Racela R, Kaufmann H. Seropositive myasthenia and autoimmune autonomic ganglionopathy: cross reactivity or subclinical disease? Auton Neurosci. 2011;164:87-8.

18 Mantegazza R, Meisel A, Sieb JP, Le Masson G, Desnuelle C, Essing M. The European LEMS Registry: baseline demographics and treatment approaches. Neurol Ther. 2015;4:105-24.

19 Sakakibara R, Uchiyama T, Tamura N, Kuwabara S, Asahina M, Hattori T. Urinary retention and sympathetic sphincter obstruction in axonal Guillain-Barré syndrome. Muscle Nerve. 2007;35:111-5.

20 Staskin D, Frankel J, Varano S, Shortino D, Jankowich R, Mudd PN Jr. International phase III, randomized, double-blind, placebo and active controlled study to evaluate the safety and efficacy of vibegron in patients with symptoms of overactive bladder: EMPOWUR. J Urol. 2020 Feb 18;204:316.

21 Zozikov B, Kunchev SI, Varlev C. Application of clenbuterol in the treatment of urinary incontinence. Int Urol Nephrol. 2001;33:413-6.

22 Tateno F, Sakakibara R, Sugiyama M, Kishi M, Ogawa E, Takahashi O, et al. Lower urinary tract function in spinocerebellar ataxia 6. Low Urin Tract Symptoms. 2012;4:41-4.

23 Ito T, Sakakibara R, Shimizu E, Kishi M, Tsuyuzaki Y, Tateno F, et al. Is major depression a risk for bladder, bowel, and sexual dysfunction? Low Urin Tract Symptoms. 2012;4:87-95. 